\title{
Modeling and control of hysteresis in magnetostrictive actuators ${ }^{\text {th }}$
}

\author{
Xiaobo Tan ${ }^{\mathrm{a}, *}$, John S. Baras ${ }^{\mathrm{a}, \mathrm{b}}$ \\ ${ }^{a}$ Institute for Systems Research, University of Maryland, College Park, MD 20742, USA \\ ${ }^{\mathrm{b}}$ Department of Electrical and Computer Engineering, University of Maryland, College Park, MD 20742, USA
}

Received 17 December 2002; received in revised form 20 January 2004; accepted 5 April 2004

\begin{abstract}
A novel dynamic model is proposed for the hysteresis in magnetostrictive actuators by coupling a Preisach operator to an ordinary differential equation, and a parameter identification method is described. An efficient inversion algorithm for a class of Preisach operators with piecewise uniform density functions is then introduced, based upon which an inverse control scheme for the dynamic hysteresis model is presented. Finally the inversion error is quantified and $l_{1}$ control theory is applied to improve the robustness of inverse compensation. Simulation and experimental results based on a Terfenol-D actuator are provided.
\end{abstract}

(C) 2004 Elsevier Ltd. All rights reserved.

Keywords: Hysteresis; Magnetostrictive actuator; Preisach operator; Inverse compensation; Robust control

\section{Introduction}

Magnetostriction is the phenomenon of strong coupling between magnetic properties and mechanical properties of some ferromagnetic materials: strains are generated in response to an applied magnetic field, while conversely, mechanical stresses in the materials produce measurable changes in magnetization. This phenomenon can be used for actuation and sensing. Fig. 1 shows a schematic of a Terfenol-D actuator manufactured by Etrema Products Inc. The magnetic field generated by the coil current controls the strain in the Terfenol-D rod, which translates into displacement or force (if blocked) output of the actuator.

Like other smart materials (e.g., piezoelectrics and shape memory alloys), magnetostrictives display strong hysteresis, which makes their effective use quite challenging. Modeling and control of hysteresis in smart materials have attracted increasing attention in recent years (Moheimani \& Goodwin, 2001). Hysteresis models can be roughly classified into

\footnotetext{
th This paper was not presented at any IFAC meeting. This was recommended for publication in revised form by Associate Editor Keum-Shik Hong under the direction of Editor Mituhiko Araki.

* Corresponding author. Present address: Department of Electrical and Computer Engineering, Michigan State University, East Lansing, MT 48824, USA. Fax: +1-517-3531980.

E-mail addresses: xbtan@egr.msu.edu (X. Tan), baras@isr.umd.edu (J.S. Baras).
}

physics-based models and phenomenological models. Physics-based models are built on first principles of physics, an example of which is the Jiles-Atherton model of ferromagnetic hysteresis (Jiles \& Atherton, 1986). Phenomenological models, on the other hand, are used to produce behaviors similar to those of physical systems without necessarily providing physical insight into the problems. A popular phenomenological hysteresis model adopted for smart materials is the Preisach model (Adly, Mayergoyz \& Bergqvis, 1991; Hughes \& Wen, 1994; Ge \& Jouaneh, 1996; Gorbet, Wang, \& Morris, 1998; Cruz-Hernandez \& Hayward, 2001; Tan, Venkataraman, \& Krishnaprasad, 2001; Natale, Velardi, \& Visone, 2001; Croft, Shed, \& Devasia, 2001), where the hysteresis is modeled as a (weighted) aggregate effect of all possible delayed relay elements. A similar operator using delayed relays of finite slopes, called Krasnosel'skii -Pokrovskii (KP) operator, has also been used ( Banks, Kurdila, \& Webb, 1997; Galinaitis \& Rogers, 1998).

The hysteretic behavior between the current input and the displacement output of a magnetostrictive actuator is rate-dependent, i.e., it depends on how fast the current is varied (see the solid-line curves in Fig. 7). However, the classical Preisach operator is rate-independent. "Dynamic" generalizations of the Preisach operator were proposed by assuming output-rate-dependent Preisach density functions (Mayergoyz, 1991), or input-rate-dependent behavior of delayed relays (Bertotti, 1992). Mrad and $\mathrm{Hu}$ (2002) proposed 


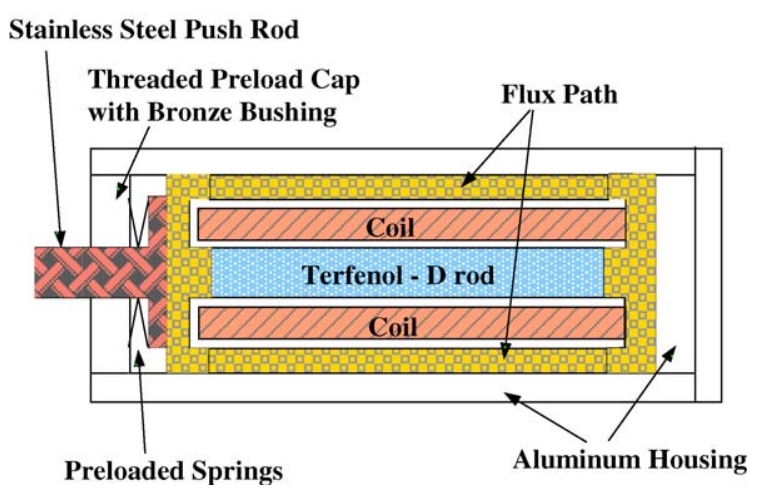

Fig. 1. Sectional view of a Terfenol-D actuator manufactured by Etrema.

a dynamic hysteresis model for piezoceramic actuators by assuming an average-input-rate-dependent Preisach density function.

Eddy current losses and magnetoelastic dynamics of the magnetostrictive rod were considered to be the origin of the rate-dependent hysteresis in Venkataraman (1999), where the eddy current losses were modeled by placing a resistor in parallel with a hysteretic inductor and the magnetoelastic dynamics was modeled by a second-order linear system. Considering a low-dimensional ferromagnetic hysteresis model led to an overall model for magnetostrictive actuators described by a set of switching ordinary differential equations (ODEs) (Venkataraman, 1999). In Venkataraman and Krishnaprasad (2000) the authors suggested using a cascade of a Preisach operator with a linear system to model magnetostrictive actuators. In this paper, based upon the model structure in Venkataraman (1999) but with a Preisach operator representing the ferromagnetic hysteresis, a novel dynamic model is proposed. This model features an unusual coupling of a Preisach operator to an ODE, which cannot be simply decomposed as a cascade of the Preisach operator with a linear system. Parameter identification methods for this model are discussed. Comparison with experimental measurements shows that the model captures the dynamic and hysteretic behavior of magnetostrictive actuators.

Inverse compensation is a fundamental approach to coping with hysteresis, where one aims to cancel out the hysteresis effect by constructing a right inverse of the hysteresis operator, see, e.g., Hughes and Wen (1994); Tao and Kokotovic (1995); Smith (1998). Inversion of the Preisach operator has been extensively studied, and general inversion algorithms are computation-intensive and/or storage-intensive (Hughes \& Wen, 1994; Reimers \& Torre, 1998; Venkataraman \& Krishnaprasad, 2000; Natale et al., 2001). The second contribution of this paper is the development of an efficient inversion algorithm for a class of Preisach operators with piecewise constant Preisach densities, and its application to inversion of the dynamic hysteresis model. Such operators arise naturally as approximations to Preisach operators with arbitrary Preisach densities.
The performance of open-loop inverse compensation is susceptible to model uncertainties and to errors introduced by (inexact) inverse algorithms. To combat this problem, one approach is adaptive inverse control (Tao \& Kokotovic, 1995; Webb et al., 1998; Kuhnen \& Janocha, 1999). In this paper we explore an alternative approach and develop a robust control framework for magnetostrictive actuators. It is shown that, for both the Preisach operator and the dynamic hysteresis model, the inversion error can be bounded in magnitude and the bound is quantifiable in terms of parameter uncertainties and the inversion scheme. Hence one can model the inversion error as an exogenous disturbance and attenuate its impact by $l_{1}$ control techniques. A systematic controller design method is presented which provides robust stability and robust trajectory tracking while taking the actuator saturation into account.

The remainder of the paper is organized as follows. The dynamic hysteresis model together with its identification method is presented in Section 2. Inversion algorithms for the Preisach operator and the dynamic hysteresis model are discussed in Section 3. The robust control framework is developed in Section 4. Finally, conclusions and discussion are provided in Section 5.

\section{A dynamic hysteresis model for magnetostrictive actuators}

\subsection{The preisach operator}

The Preisach operator is briefly reviewed in this subsection to fix the notation and provide the background for later developments. A detailed treatment can be found in Mayergoyz (1991); Visintin (1994); Brokate and Sprekels (1996). For a pair of thresholds $(\beta, \alpha)$ with $\beta \leqslant \alpha$, consider a delayed relay $\hat{\gamma}_{\beta, \alpha}[\cdot, \cdot]$, as illustrated in Fig. 2. For $u \in C([0, T])$ (the space of continuous functions on $[0, T]$ ) and an initial configuration $\zeta \in\{-1,1\}, \omega=\hat{\gamma}_{\beta, \alpha}[u, \zeta]$ is defined as, for $t \in[0, T]$,

$\omega(t) \triangleq\left\{\begin{array}{ll}-1 & \text { if } u(t)<\beta, \\ 1 & \text { if } u(t)>\alpha, \\ \omega\left(t^{-}\right) & \text {if } \beta \leqslant u(t) \leqslant \alpha,\end{array}\right.$,

where $\omega\left(0^{-}\right)=\zeta$ and $t^{-} \triangleq \lim _{\varepsilon>0, \varepsilon \rightarrow 0} t-\varepsilon$.

Define the Preisach plane

$\mathscr{P}_{0} \triangleq\left\{(\beta, \alpha) \in \mathbb{R}^{2}: \beta \leqslant \alpha\right\}$,

where $(\beta, \alpha) \in \mathscr{P}_{0}$ is identified with $\hat{\gamma}_{\beta, \alpha}$. For $u \in C([0, T])$ and a Borel measurable configuration $\zeta_{0}$ of all delayed relays, $\zeta_{0}: \mathscr{P}_{0} \rightarrow\{-1,1\}$, the output of the Preisach operator $\Gamma$ is defined as

$\Gamma\left[u, \zeta_{0}\right](t)=\int_{\mathscr{P}_{0}} \hat{\gamma}_{\beta, \alpha}\left[u, \zeta_{0}(\beta, \alpha)\right](t) \mathrm{d} v(\beta, \alpha)$,

where $v$ is a finite Borel measure on $\mathscr{P}_{0}$, called the Preisach measure. In this paper $v$ is called nonsingular if $|v|$ is 


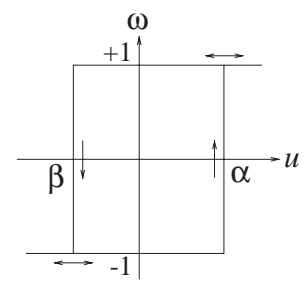

Fig. 2. A delayed relay $\hat{\gamma}_{\beta, \alpha}[\cdot, \cdot]$.

absolutely continuous with respect to the two-dimensional Lebesgue measure. For a nonsingular v, (1) can be rewritten as

$\Gamma\left[u, \zeta_{0}\right](t)=\int_{\mathscr{P}_{0}} \mu(\beta, \alpha) \hat{\gamma}_{\beta, \alpha}\left[u, \zeta_{0}(\beta, \alpha)\right](t) \mathrm{d} \beta \mathrm{d} \alpha$,

for some Borel measurable function $\mu$, called the Preisach density function. It is assumed in this paper that $\mu$ has a compact support

$\mathscr{P} \triangleq\left\{(\beta, \alpha) \in \mathscr{P}_{0} \mid \beta \geqslant \beta_{0}, \alpha \leqslant \alpha_{0}\right\}$,

where $\alpha_{0}=-\beta_{0}=s_{0}$ for some $s_{0}>0 . \mathscr{P}$ is also called the Preisach plane when no confusion arises.

At any time $t, \mathscr{P}$ is a disjoint union of two sets, $\mathscr{P}_{+}(t)$ and $\mathscr{P}_{-}(t)$, where $\mathscr{P}_{+}(t)\left(\mathscr{P}_{-}(t)\right.$, resp. $)$ consists of points $(\beta, \alpha)$ such that the output of $\hat{\gamma}_{\beta, \alpha}$ at $t$ is $+1(-1$, resp.). Under mild conditions, each of $\mathscr{P}_{+}(t)$ and $\mathscr{P}_{-}(t)$ is a connected set, and the boundary between them, called the memory curve, characterizes the memory of the Preisach operator. The memory curve has a staircase structure and the coordinates of its intersection with the line $\alpha=\beta$ correspond to the current value of the input. The set of all memory curves is denoted as $\Psi$. The memory curve $\psi_{0}$ at $t=0$ is called the initial memory curve and hereafter it will be put as the second argument of $\Gamma$. The following theorem collects several properties of the Preisach operator that are relevant to this paper:

Theorem 2.1 (Visintin, 1994). Let $u \in C([0, T])$ and $\psi_{0} \in \Psi$.

(1) (Rate independence) If $\phi:[0, T] \rightarrow[0, T]$ is an increasing continuous function such that $\phi(0)=0$ and $\phi(T)=T$, then

$$
\Gamma\left[u \circ \phi, \psi_{0}\right](t)=\Gamma\left[u, \psi_{0}\right](\phi(t)), \quad \forall t \in[0, T],
$$

where "o" denotes composition of functions.

(2) (Piecewise Monotonicity) Let $v \geqslant 0$. If $u$ is either nondecreasing or nonincreasing on some interval in $[0, T]$, then so is $\Gamma\left[u, \psi_{0}\right]$.

(3) (Lipschitz Continuity) Let $v \geqslant 0$ and

$$
v(\mathscr{N}(\psi, \varepsilon)) \leqslant C_{v} \varepsilon, \quad \forall \psi \in \Psi, \quad \forall \varepsilon>0,
$$

where $\mathscr{N}(\psi, \varepsilon)$ denotes the $\varepsilon$-neighborhood of $\psi$. Then $\Gamma$ is Lipschitz continuous with Lipschitz constant $2 C_{v}$.

In identification of the Preisach measure, a discretization step is involved in one way or another (see Tan (2002)

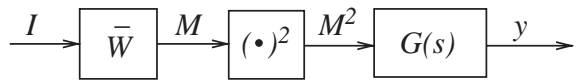

Fig. 3. Model structure of a magnetostrictive actuator.

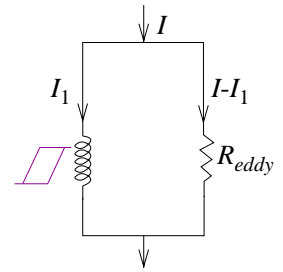

Fig. 4. Representation of eddy current losses.

for a review of identification methods). One discretization scheme is to divide the input range into $L$ intervals uniformly (called discretization of level $L$ ), which results in a discretization grid on the Preisach plane. An arbitrary Preisach density function can then be approximated by one that is constant within each discretization cell. Note that this type of approximation enjoys nice convergence properties (Hoffmann et al., 1988). To obtain such a piecewise constant approximation to an unknown Preisach density function, one can first identify the weighting masses for the discretization cells using a constrained least squares algorithm (Tan et al., 2001), and then distribute each mass uniformly over the corresponding cell.

\subsection{The dynamic hysteresis model}

The model for a magnetostrictive actuator has a cascaded structure as shown in Fig. 3, where $I, M$ and $y$ denote the current input, the bulk magnetization along the rod direction, and the displacement output, respectively. The block $\bar{W}$ takes care of the ferromagnetic hysteresis and the eddy current losses as illustrated in Fig. 4, and $G(s)$ is a lumped model for the magnetoelastic dynamics.

The voltage $V$ across the nonlinear inductor is $N_{m} A_{m} \frac{\mathrm{d} B}{\mathrm{~d} t}$, where $B$ is the magnetic flux density, $N_{m}$ is the number of turns of the coil, and $A_{m}$ is the cross-sectional area of the rod. Since $V=\left(I-I_{1}\right) R_{\text {eddy }}$,

$\frac{\mathrm{d} B}{\mathrm{~d} t}=\frac{R_{\text {eddy }}}{N_{m} A_{m}}\left(I-I_{1}\right)$.

In SI units, $B=\mu_{0}(H+M)$, where $\mu_{0}=4 \pi \times 10^{-7} \mathrm{Henry} / \mathrm{m}$ is the permeability of vacuum and $H$ is the magnetic field along the rod direction. $H$ is related to $I_{1}$ via $H=c_{0} I_{1}+H_{\text {bias }}$, where $c_{0}$ is the coil factor and $H_{\text {bias }}$ is the bias field necessary for generating bi-directional strains. Letting $M=\Gamma\left[H, \psi_{0}\right]$, one obtains the dynamic hysteresis model:

$$
\left\{\begin{array}{l}
\dot{H}(t)+\dot{M}(t)=c_{1}\left(I(t)-\frac{H(t)-H_{\text {bias }}}{c_{0}}\right), \\
M(t)=\Gamma\left[H(\cdot), \psi_{0}\right](t),
\end{array},\right.
$$

where $c_{1} \triangleq \frac{R_{\text {eddy }}}{\mu_{0} N_{m} A_{m}}$. 


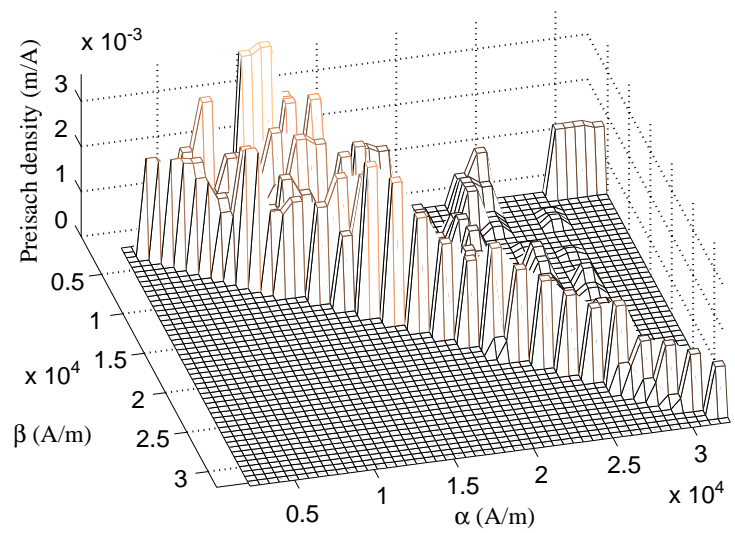

Fig. 5. A piecewise constant Preisach density function.

The following theorem shows that (4) is well-posed, the proof of which can be found in Tan (2002).

Theorem 2.2. If the Preisach measure $v$ is nonnegative and nonsingular, and $I(\cdot)$ is piecewise continuous, then $\forall \psi_{0} \in \Psi, \forall T>0$, there exists a unique pair $\{H(\cdot), M(\cdot)\} \in C([0, T]) \times C([0, T])$ satisfying (4) almost everywhere.

$G(s)$ has a state space representation:

$\ddot{y}(t)+2 \xi \omega_{0} \dot{y}(t)+\omega_{0}^{2} y(t)=\frac{\omega_{0}^{2} l_{\text {rod }} \lambda_{s}}{M_{s}^{2}} M^{2}(t)$,

where $\omega_{0}=2 \pi f_{0}, f_{0}$ is the first resonant frequency of the actuator, $\xi$ is the damping coefficient, $l_{\text {rod }}$ is the rod length, $\lambda_{\mathrm{s}}$ is the saturation magnetostriction and $M_{\mathrm{s}}$ is the saturation magnetization.

\subsection{Identification of model parameters}

The magnetostrictive actuator used in this study is an AA-050H series Terfenol-D actuator (full operating range is about $50 \mu \mathrm{m}$ ) manufactured by Etrema. The current input is provided by a Kepco power supply (Model BOP 36-6M) operating in Current Programming mode, which is controlled by a Pentium III $450 \mathrm{MHz}$ PC with a DSpace DS1103 PPC Controller Board. The displacement of the actuator is measured with an LVDT sensor (Schaevitz 025MHR).

The following parameters are available from the manufacturer: $N_{m}=1300, A_{m}=2.83 \times 10^{-5} \mathrm{~m}^{2}, c_{0}=1.54 \times$ $10^{4} / \mathrm{m}, M_{s}=7.87 \times 10^{5} \mathrm{~A} / \mathrm{m}, l_{\text {rod }}=5.13 \times 10^{-2} \mathrm{~m}$, and the following parameters can be identified relatively easily: $\lambda_{s}=1.313 \times 10^{-3}, H_{\text {bias }}=1.23 \times 10^{4} \mathrm{~A} / \mathrm{m}, f_{0}=392 \mathrm{~Hz}$. A piecewise uniform Preisach density function for $\Gamma$, shown in Fig. 5, was identified using the method explained in Section 2.1, where the level of discretization $L=25$.

To identify $R_{\text {eddy }}$ and $\xi$, one first discretizes the range $[0, \bar{R}]$ for $R_{\text {eddy }}$ with the mesh points denoted as

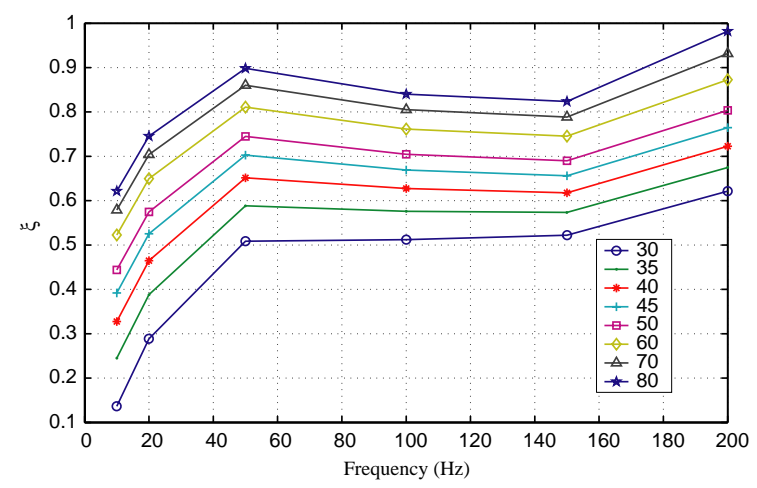

Fig. 6. Variation of $\xi_{k}^{(i)}$ with respect to the input frequency for different $R_{\text {eddy }}^{(i)}$.

$R_{\text {eddy }}^{(i)}, i=1, \ldots, N$, and then perform the following:

- Step 1: Apply a sinusoidal current (with some dc shift) $I(\cdot)$ of frequency $f$ to the actuator and measure the phase lag $\theta_{y, I}$ between the fundamental frequency component of $y(\cdot)$ and $I(\cdot)$;

- Step 2: For each $R_{\text {eddy }}^{(i)}$, numerically integrate (4) with $I(\cdot)$ as the input, and calculate the phase lag $\theta_{M^{2}, I}$ between the fundamental frequency component of $M^{2}(\cdot)$ and $I(\cdot)$;

- Step 3. The difference $\theta_{y, I}-\theta_{M^{2}, I}$ is considered to be the phase lag between the fundamental frequency component of $y(\cdot)$ and that of $M^{2}(\cdot)$ in (5), from which one can compute $\xi^{(i)}$.

Repeat the above procedure (Step 1 to Step 3 ) $K$ times with different input frequencies and denote the damping coefficients as $\left\{\xi_{k}^{(i)}\right\}_{k=1}^{K}$ for $R_{\text {eddy }}^{(i)}$. If $R_{\text {eddy }}^{(i)}$ is close to the true parameter $R_{\text {eddy }}, \xi_{k}^{(i)}$ should not vary much with $k$. Hence pick $i^{*} \in\{1, \ldots, N\}$ such that $\left\{\xi_{k}^{\left(i^{*}\right)}\right\}_{k=1}^{K}$ has the minimum variance, let $R_{\text {eddy }}=R_{\text {eddy }}^{\left(i^{*}\right)}$, and let $\xi$ be the mean of $\left\{\xi_{k}^{\left(i^{*}\right)}\right\}_{k=1}^{K}$.

The current amplitude used in identification is $0.8 \mathrm{~A}$ with a $0.1 \mathrm{~A}$ dc shift. Fig. 6 shows the variation of $\xi$ with respect to the frequency for different $R_{\text {eddy }}^{(i)}$ 's, from which it is determined that $R_{\text {eddy }}=70 \Omega, \xi=0.7783$. Fig. 7 compares the frequency-dependent hysteresis loops measured in experiments to those obtained through simulation based on the identified parameters. Good agreement is achieved up to $200 \mathrm{~Hz}$. To further verify the model, comparison is conducted at 10,20 , and $50 \mathrm{~Hz}$ for a different input range $[-0.7 A, 0.3 \mathrm{~A}]$, and good fit is also achieved (Fig. 8). Hence the model (4) and (5) is able to capture the dynamic and hysteretic behavior of magnetostrictive actuators in a reasonably wide frequency range. Beyond $200 \mathrm{~Hz}$, simulation results and experimental measurements still qualitatively agree although the fit gets worse. This indicates that further details of the eddy currents and the magnetoelastic dynamics need to be considered to fully capture the dynamic behaviors at very high frequencies. 

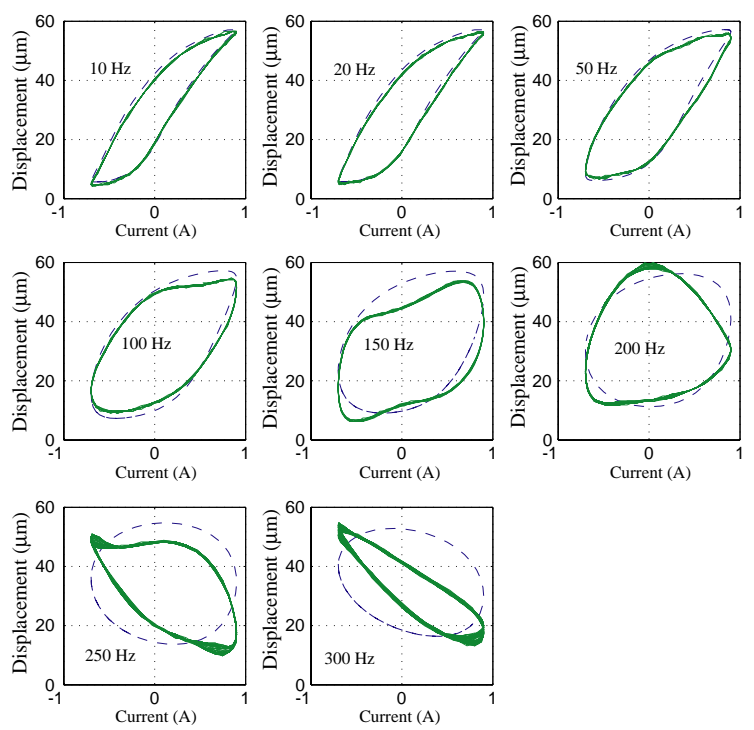

Fig. 7. Comparison of rate-dependent hysteresis loops (current input range: [ -0.7 A, 0.9 A]). Solid line: experimental measurement; Dashed line: numerical prediction.
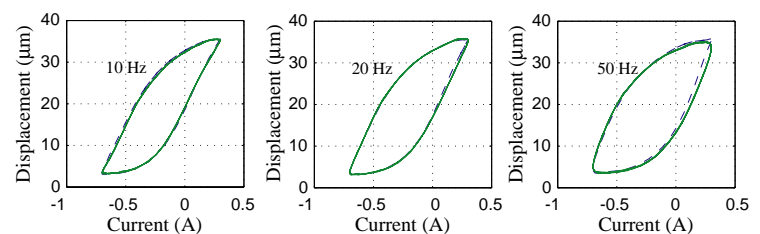

Fig. 8. Comparison of rate-dependent hysteresis loops (current input range: [ 0.7 A, 0.3 A]). Solid line: experimental measurement; Dashed line: numerical prediction.

\section{Inverse compensation algorithms}

\subsection{Inversion of the Preisach operator}

Consider a discretization scheme of level $L$ and let the Preisach density function $\mu_{p}$ be nonnegative and constant within each discretization cell. The inversion problem is, given $\psi_{0} \in \Psi$ and a value $\bar{M}$, to find $\bar{H}$ such that $\bar{M}=$ $\Gamma\left[\bar{H}, \psi_{0}\right]$.

Let the input and the output of the Preisach operator corresponding to $\psi_{0}$ be $H_{0}$ and $M_{0}$, respectively. Assuming $\bar{M}>M_{0}$ (the case $\bar{M}<M_{0}$ can be treated analogously), the following algorithm is proposed to find $\bar{H}$ :

- Step $1: H^{(0)}:=H_{0}, M^{(0)}:=M_{0}, \psi^{(0)}:=\psi_{0}, n:=0$;

- Step 2:

$$
\left\{\begin{array}{l}
d^{(n)}:=\min \left\{d_{0}^{(n)}, d_{1}^{(n)}, d_{2}^{(n)}\right\} \\
H^{(n+1)}:=H^{(n)}+d^{(n)} \\
M^{(n+1)}:=\Gamma\left[H^{(n+1)}, \psi^{(n)}\right]
\end{array}\right.
$$

where $\psi^{(n)}$ is the memory curve after $\left\{H^{(k)}\right\}_{k=1}^{n}$ is applied, and $d_{0}^{(n)}, d_{1}^{(n)}, d_{2}^{(n)}$ are determined in the follow-

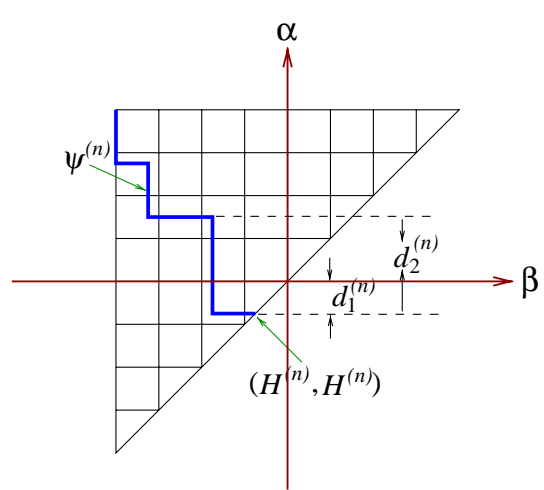

Fig. 9. Illustration of $d_{1}^{(n)}$ and $d_{2}^{(n)}$ on the Preisach plane $(L=8)$.

ing way (see Fig. 9 for illustration):

$\circ$ Let $d_{1}^{(n)}>0$ be such that $H^{(n)}+d_{1}^{(n)}$ equals the next discrete input level;

○ Let $d_{2}^{(n)}>0$ be the minimum value such that applying $H^{(n)}+d_{2}^{(n)}$ would eliminate the next corner of $\psi^{(n)}$

○ Compute $a_{1}^{(n)}, a_{2}^{(n)} \geqslant 0$ satisfying, for $0<d<$ $\min \left\{d_{1}^{(n)}, d_{2}^{(n)}\right\}$

$$
\Gamma\left[H^{(n)}+d, \psi^{(n)}\right]-\Gamma\left[H^{(n)}, \psi^{(n)}\right]=a_{2}^{(n)} d^{2}+a_{1}^{(n)} d,
$$

and let $d_{0}^{(n)}>0$ be the solution to

$$
\bar{M}-\Gamma\left[H^{(n)}, \psi^{(n)}\right]=a_{2}^{(n)}\left(d_{0}^{(n)}\right)^{2}+a_{1}^{(n)} d_{0}^{(n)} .
$$

If $d^{(n)}=d_{0}^{(n)}$, go to Step 3; otherwise let $n:=n+1$ and go to Step 2;

- Step 3: $\bar{H}:=H^{(n+1)}$ and stop.

The algorithm is based on the piecewise monotonicity property of $\Gamma$. It yields the (exact) solution in no more than $\bar{n}=n_{c}\left(\psi_{0}\right)+L$ iterations, where $n_{c}\left(\psi_{0}\right)$ is the number of corners of $\psi_{0}$.

\subsection{Inversion of the dynamic hysteresis model}

Given $\psi_{0} \in \Psi$ and a desired trajectory $\bar{M}(\cdot)$, the inversion problem for (4) is to find $I(\cdot)$, such that the corresponding output of $\bar{W}$ is $\bar{M}(\cdot)$. The following (formal) inverse scheme for (4) is proposed:

$$
\left\{\begin{array}{l}
\bar{H}(t)=\Gamma^{-1}\left[\bar{M}(\cdot), \psi_{0}\right](t) \\
I(t)=\frac{1}{c_{1}}(\dot{\bar{H}}(t)+\dot{\bar{M}}(t))+\frac{\bar{H}(t)-H_{\text {bias }}}{c_{0}}
\end{array} .\right.
$$

In implementation $\Gamma^{-1}$ is constructed with the inversion scheme presented in Section 3.1, and $\dot{\bar{M}}$ and $\dot{\bar{H}}$ are approximated by the finite difference method.

Displacement tracking experiments are conducted to further validate the model and examine the performance of the inverse scheme (7). To avoid the input saturation in (5), 

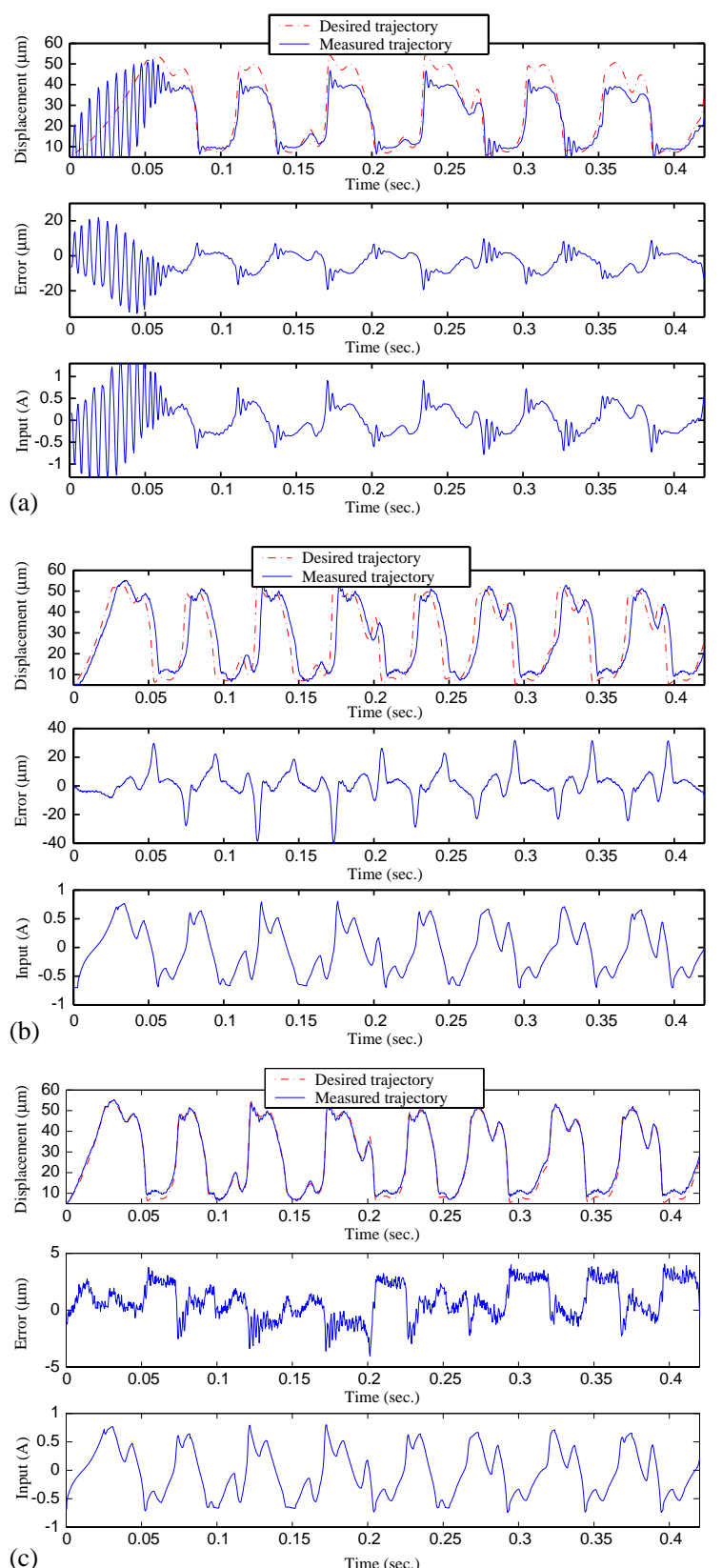

(c)

Fig. 10. Experimental results of trajectory tracking. (a) Proportional feedback control; (b) inverse compensation based on the static hysteresis model; (c) inverse compensation based on the dynamic hysteresis model.

the desired displacement trajectory $\bar{y}(\cdot)$ is picked so that

$0 \leqslant \bar{u}(t) \triangleq \frac{M_{s}^{2}}{\omega_{0}^{2} l_{\text {rod }} \lambda_{s}}\left(\ddot{\bar{y}}(t)+2 \xi \omega_{0} \dot{\bar{y}}(t)+\omega_{0}^{2} \bar{y}(t)\right) \leqslant M_{s}^{2}$,

in which case $\bar{M}(\cdot)$ is computed as: $\forall t, \bar{M}(t)=\sqrt{\bar{u}(t)}$.

The inverse control scheme (7) is compared with a proportional feedback scheme and an inversion scheme based on a static hysteresis model (which is essentially a Preisach operator), see Fig. 10(a)-(c). In each figure, the displacement trajectories (both the desired and the measured), the tracking error, and the current applied are displayed. Al- though the controller parameter has been carefully tuned, the performance of the pure feedback scheme is poor (Fig. 10(a)). This highlights the need for hysteresis compensation. Under the static model-based inverse compensation, the achieved trajectory displays an appreciable phase lag with respect to the desired one (Fig. 10(b)). On the other hand, the inverse scheme based on the dynamic model delivers satisfactory performance, and almost perfect tracking is achieved for the full operating range of the actuator (Fig. 10(c)).

\section{A robust control framework}

Models for smart material actuators have a cascaded structure as shown in Fig. 11(a). Here $W$ is a hysteretic operator which could be rate-independent or rate-dependent. $\hat{G}_{a}(\lambda)$ denotes the $\lambda$-transform of a discrete-time, linear time-invariant system $G_{a}{ }^{1}$, and represents the linear part of the actuator dynamics. Note the resemblance of Fig. 11(a) with Fig. 3. In Fig. 11(b), $\hat{G}_{0}(\lambda)$ denotes the plant to be controlled by the actuator, and $\tilde{W}^{-1}$ denotes the approximate right inverse of $W$. The goal is to design the controller $\hat{K}(\lambda)$ which guarantees the closed-loop system stability and minimizes the tracking error in the presence of the inversion error $e_{u}=\tilde{u}-u$ and the uncertainties in $\hat{G}_{a}$ and $\hat{G}_{0}$. It is also desired to meet the saturation constraint in the controller design.

\subsection{Quantification of the inversion error}

The error in inversion of the Preisach operator and the dynamic hysteresis model are quantified respectively next. The signal space used is $l_{\infty}$, the space of sequences of bounded magnitude.

\subsubsection{Error in inversion of the Preisach operator}

Let $v$ be a nonsingular, nonnegative measure with density $\mu$. For a discretization scheme of level $L$, let $v_{p}$ be a piecewise uniform approximation to $v$ obtained as described in Section 2.1. When several Preisach operators are involved in the discussion, the corresponding Preisach measure will be put as the subscript of $\Gamma$ to avoid confusion. Given a desired sequence $\bar{M}[\cdot] \in l_{\infty}$ and $\psi_{0} \in \Psi$, let

$H[k]=\Gamma_{v_{p}}^{-1}\left[\bar{M}[\cdot], \psi_{0}\right][k], \quad \forall k \geqslant 0$,

where $\Gamma_{v_{p}}^{-1}$ is the (exact) inverse of $\Gamma_{v_{p}}$ constructed as in Section 3.1. Let $\tilde{M}=\Gamma_{v}\left[H[\cdot], \psi_{0}\right]$ and define the inversion error $e_{M}[\cdot]=\tilde{M}[\cdot]-\bar{M}[\cdot]$.

Proposition 4.1. Let $\mu \leqslant \bar{\mu}$ for some constant $\bar{\mu}$. Denote the integral of $\mu$ over discretization cell $i$ as $v_{i}^{0}, 1 \leqslant i \leqslant N_{c}$, where $N_{c}$ is the number of cells. Denote by $v_{i}$ the identified

\footnotetext{
${ }^{1}$ The $\lambda$-transform $\hat{G}(\lambda)$ is just the usual $z$-transform of $G$ with $\lambda=z^{-1}$ (Dahleh \& Diaz-Bobillo, 1995).
} 
(a)
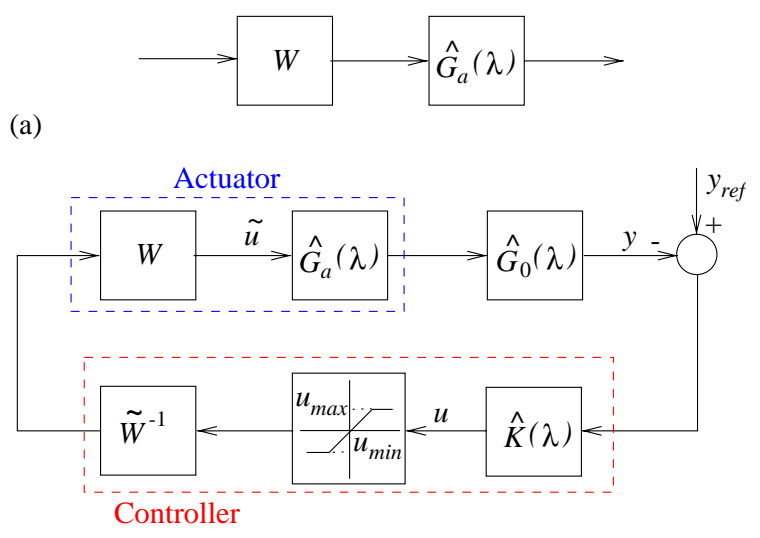

(b)

Fig. 11. (a) A model structure for smart actuators; (b) the closed-loop system incorporating inverse compensation.

Preisach weighting mass for cell $i$. Assume that the relative error in identification is $\delta_{I}$, i.e., $\frac{\left|v_{i}-v_{i}^{0}\right|}{v_{i}^{0}} \leqslant \delta_{I}, 1 \leqslant i \leqslant N_{c}$. Then $\left\|e_{M}\right\|_{\infty} \leqslant \delta_{I} M_{s}+\frac{8 \bar{\mu} s_{0}^{2}}{L}$, where $M_{s}$ is the saturation output of $\Gamma_{v}$, and $s_{0}$ is as defined in Section 2.1.

Proof. Let $v_{p}^{0}$ be another piecewise constant Preisach measure obtained by distributing $v_{i}^{0}$ uniformly over the cell $i$, $1 \leqslant i \leqslant N_{c}$. For any $k \geqslant 0$,

$$
\begin{aligned}
\left|e_{M}[k]\right|= & \left|\Gamma_{v}\left[H[\cdot], \psi_{0}\right][k]-\Gamma_{v_{p}}\left[H[\cdot], \psi_{0}\right][k]\right| \\
\leqslant & \left|\Gamma_{v}\left[H[\cdot], \psi_{0}\right][k]-\Gamma_{v_{p}^{0}}\left[H[\cdot], \psi_{0}\right][k]\right| \\
& +\left|\Gamma_{v_{p}^{0}}\left[H[\cdot], \psi_{0}\right][k]-\Gamma_{v_{p}}\left[H[\cdot], \psi_{0}\right][k]\right| .
\end{aligned}
$$

All three Preisach operators involved in (8) share the same memory curve $\psi[k], \forall k \geqslant 0$. It is clear that the second term of (8) is bounded by $\delta_{I} M_{s}$. To bound the first term, note that for any $k \geqslant 0, \psi[k]$ spans $L-1$ square cells and one triangular cell. Any cell not touched by $\psi[k]$ contributes the same to $\Gamma_{v}\left[H[\cdot], \psi_{0}\right][k]$ and $\Gamma_{v_{p}^{0}}\left[H[\cdot], \psi_{0}\right][k]$. Hence the first term of (8) is bounded by twice the interval of $\mu$ over cells spanned by $\psi[k]$, which is further bounded by $\frac{2 \bar{\mu}\left(2 s_{0}\right)^{2}(L-1 / 2)}{L^{2}}<\frac{8 \bar{\mu} s_{0}^{2}}{L}$.

From Proposition 4.1, the bound on the inversion error consists of two parts: the first part is proportional to the relative identification error, and the second part is inversely proportional to the level $L$ of discretization.

\subsubsection{Error in inversion of the dynamic hysteresis model}

For the dynamic hysteresis model, the inversion error based on scheme (7) is hard to quantify. Hence another inversion algorithm is introduced here. When the Preisach density $\mu$ is piecewise continuous, (4) can be rewritten as:

$$
\left\{\begin{array}{l}
\dot{H}(t)=\frac{c_{1}}{1+g(t)}\left(I(t)-\frac{H(t)-H_{\text {bias }}}{c_{0}}\right) \\
M(t)=\Gamma\left[H(\cdot), \psi_{0}\right](t)
\end{array},\right.
$$

where $g(t)$ carries the interpretation of " $\frac{\mathrm{d} M}{\mathrm{~d} H}(t)$ ", and $0 \leqslant g(t) \leqslant C_{g}$ for some constant $C_{g}>0$. Eq. (9) can be viewed as perturbed from a decoupled system obtained by replacing $g(t)$ with some constant $\bar{g} \in\left[0, C_{g}\right]$. Based on the decoupled system, an approximate inversion scheme for (9) and thus for (4) is given by (in discrete-time)

$$
\left\{\begin{array}{l}
\bar{H}[k]=\Gamma^{-1}\left[\bar{M}[\cdot], \psi_{0}\right][k], \\
I[k]=\frac{1+\bar{g}}{c_{1}} \frac{\bar{H}[k]-\bar{H}[k-1]}{h}+\frac{\bar{H}[k-1]-H_{\text {bias }}}{c_{0}},
\end{array}\right.
$$

where $h$ is the time step. In (10) the finite forward difference of $\bar{H}[\cdot]$ is used to approximate the continuous time derivative. Apply $I[\cdot]$ in (10) to the finite forward difference implementation of (9), and denote the corresponding solution as $\tilde{H}[\cdot]$ and $\tilde{M}[\cdot]$. The inversion error $e_{M}[\cdot]$ is now defined as $e_{M}[k] \triangleq \tilde{M}[k]-\bar{M}[k-1]$, where the delay is introduced due to the dynamics in (9). The following result quantifies $\left\|e_{M}\right\|_{\infty}$ assuming that parameters are exact and $\Gamma^{-1}$ is constructed perfectly in (10).

Proposition 4.2. Let the Preisach measure $v$ be nonnegative and nonsingular with a piecewise continuous density, and $\Gamma$ be Lipschitz continuous with Lipschitz constant $C_{v}$. Let $\bar{H}[-1]=\bar{H}[0]=\tilde{H}[0]$. Pick $\bar{g} \in\left[0, C_{g}\right]$. Then $\forall \bar{M}[\cdot] \in l_{\infty}, \forall \psi_{0} \in \Psi$,

$\left\|e_{M}\right\|_{\infty} \leqslant 2 C_{v} \bar{\gamma}_{1} s_{0}$

where $\bar{\gamma}_{1}=\frac{\max \left\{\bar{g}, \frac{C_{g}-\bar{g}}{1+C_{g}}\right\}}{1-\max \left\{\frac{h c_{1}}{c_{0}}-1,1-\frac{h c_{1}}{c_{0}\left(1+C_{g}\right)}\right\}}$. The optimal $\bar{g}$ to minimize $\bar{\gamma}_{1}$ is $\frac{C_{g}}{C_{g}+2}$.

Proof. Define $e_{H}[k] \triangleq \tilde{H}[k]-\bar{H}[k-1], k \geqslant 0$. One can verify that $e_{H}$ satisfies:

$e_{H}[k+1]=a[k] e_{H}[k]+b[k](\bar{H}[k]-\bar{H}[k-1])$,

where $a[k] \triangleq 1-\frac{h c_{1}}{c_{0}(1+g[k])}, b[k] \triangleq \frac{\bar{g}-g[k]}{1+g[k]}$, and $g[k] \triangleq$ $g(k h)$. From $(12), e_{H}[k+1]=$

$$
\begin{aligned}
\left(\prod_{i=0}^{k} a[i]\right) e_{H}[0]+\sum_{i=0}^{k} \\
\left(\prod_{j=i+1}^{k} a[j]\right) b[i](\bar{H}[i]-\bar{H}[i-1]),
\end{aligned}
$$

which implies (noting that $e_{H}[0]=0$ )

$$
\begin{aligned}
\left|e_{H}[k+1]\right| & \leqslant 2\left(\sum_{i=0}^{k} \bar{a}^{i}\right) \bar{b}\|\bar{H}\|_{\infty} \\
& \leqslant \frac{2 \bar{b}}{1-\bar{a}}\|\bar{H}\|_{\infty},
\end{aligned}
$$




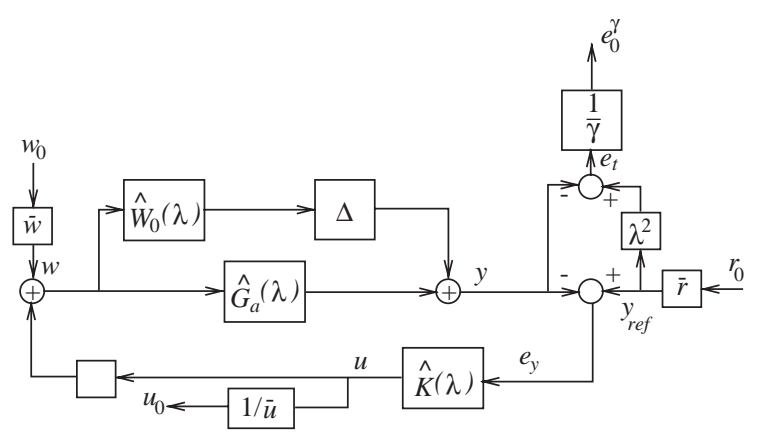

Fig. 12. Formulation of the robust control problem for a magnetostrictive actuator.

where $\bar{a} \triangleq \max _{x \in\left[0, C_{g}\right]}\left|1-\frac{h c_{1}}{c_{0}(1+x)}\right|, \bar{b} \triangleq \max _{x \in\left[0, C_{g}\right]}\left|\frac{\bar{g}-x}{1+x}\right|$. Values of $\bar{a}$ and $\bar{b}$ can be easily determined:

$\bar{a}=\max \left\{\frac{h c_{1}}{c_{0}}-1,1-\frac{h c_{1}}{c_{0}\left(1+C_{g}\right)}\right\}$,

$\bar{b}=\max \left\{\bar{g}, \frac{C_{g}-\bar{g}}{1+C_{g}}\right\}$.

Therefore $\left\|e_{H}\right\|_{\infty} \leqslant 2 \bar{\gamma}_{1}\|\bar{H}\|_{\infty}$. Since $\|\bar{H}\|_{\infty} \leqslant s_{0}$, Eq. (11) now follows using the Lipschitz continuity and the time invariance properties of $\Gamma$. It's easy to see that the optimal $\bar{g}$ minimizing $\bar{\gamma}_{1}$ is $\frac{C_{g}}{C_{g}+2}$.

Similarly one can derive the bound on inversion error when the finite backward difference method is used to approximate the time derivatives:

Proposition 4.3. Let the assumptions in Proposition 4.2 hold. Consider the finite backward difference method. Then for any $\bar{M}[\cdot] \in l_{\infty}$, for any $\psi_{0} \in \Psi$,

$\left\|e_{M}\right\|_{\infty} \leqslant 2 C_{v} \bar{\gamma}_{2} s_{0}$

where $\bar{\gamma}_{2}=\max \left\{\frac{\bar{g}}{1+\frac{c_{1} h}{c_{0}}}, \frac{C_{g}-\bar{g}}{1+C_{g}+\frac{c_{1} h}{c_{0}}}\right\} \frac{c_{0}\left(1+C_{g}\right)+c_{1} h}{c_{1} h}$. The optimal $\bar{g}$ to minimize $\bar{\gamma}_{2}$ is $\frac{\left(c_{0}+c_{1} h\right) C_{g}}{2\left(c_{0}+c_{1} h\right)+c_{0} C_{g}}$.

Note that the backward difference method is preferred in general due to its stability property. Propositions 4.2 and 4.3 quantify the errors solely due to inversion algorithms. Similar arguments can extend the error estimates to the case where there are parametric uncertainties in $c_{0}$ and $c_{1}$. The error in $\Gamma^{-1}$ can be included using Proposition 4.1. Finally $\left\|e_{u}\right\|_{\infty}$ can be derived from $\left\|e_{M}\right\|_{\infty}$ when the square nonlinearity is considered.

\subsection{Formulation of the robust control problem}

Fig. 12 shows the closed-loop system after the inverse compensation is done, where trajectory tracking of the magnetostrictive actuator is considered. The exogenous noise $w$ represents the inversion error, $\|w\|_{\infty} \leqslant \bar{w}$, and $\bar{w}$ is quantifiable in terms of inverse schemes and parametric uncertainties. $\hat{G}_{a}(\lambda)$ stands for the discretized version of $G(s)$ in Fig. 3. $\Delta \circ \hat{W}_{0}(\lambda)$ represents the uncertainty in $\hat{G}_{a}(\lambda)$, where $\Delta$ is a nonlinear uncertainty with $\|\Delta\|_{l_{\infty}-\text { ind }}<1$ and $\hat{W}_{0}(\lambda)$ is a frequency weighting function.

Let $\left\|y_{\text {ref }}\right\|_{\infty} \leqslant \bar{r}$, where $y_{\text {ref }}$ is the reference trajectory. The error $e_{y} \triangleq y_{\text {ref }}-y$ is fed into the controller $\hat{K}(\lambda)$. The delay $\lambda$ following $\hat{K}(\lambda)$ is due to inversion of (4), and another delay is contained in $\hat{G}_{a}$. Hence the tracking error is defined as $e_{t}[k] \triangleq y_{\text {ref }}[k-2]-y[k]$. Given $\gamma>0$, define $e_{0}^{\gamma} \triangleq \frac{e_{t}}{\gamma}$. To ease the formulation, $w$ and $y_{\text {ref }}$ are also normalized so that $\left\|w_{0}\right\|_{\infty} \leqslant 1,\left\|r_{0}\right\|_{\infty} \leqslant 1$ (see Fig. 12). Symmetric input saturation $|u| \leqslant \bar{u}$ translates into $\left\|u_{0}\right\|_{\infty} \leqslant 1$, where $u_{0} \triangleq \frac{u}{\bar{u}}$. Asymmetric saturation can be accommodated by letting $\bar{u}=\frac{u_{\max }-u_{\min }}{2}$ and adding a constant $u_{b}=\frac{u_{\max }+u_{\min }}{2}$ to the control.

The objective of controller design is to find the smallest $\gamma^{*}$ and a stabilizing controller $\hat{K}(\lambda)$, such that

(1) (Robust stability) the closed-loop system is stable for any $\Delta$ with $\|\Delta\|_{l_{\infty}-\text { ind }}<1$,

(2) (Tracking performance) $\left\|e_{0}^{\gamma^{*}}\right\|_{\infty} \leqslant 1$ if $\Delta=0, \forall w_{0}, r_{0}$ with $\left\|w_{0}\right\|_{\infty} \leqslant 1$ and $\left\|r_{0}\right\|_{\infty} \leqslant 1$, and

(3) (Saturation constraint) $\left\|u_{0}\right\|_{\infty} \leqslant 1$ if $\Delta=0, \forall w_{0}, r_{0}$ with $\left\|w_{0}\right\|_{\infty} \leqslant 1$ and $\left\|r_{0}\right\|_{\infty} \leqslant 1$.

Note that $\hat{K}$ will be dependent on $\gamma^{*}$ although the dependence is suppressed in the notation. Item (2) implies that the $l_{1}$ norm of the closed-loop mapping from the inputs $\left\{w_{0}, r_{0}\right\}$ to the tracking error $e_{t}$ is less than $\gamma^{*}$. Hence $\gamma^{*}$ will be called the optimal disturbance attenuation level. Design of $\hat{K}$ is a standard $l_{1}$ robust control problem, which can be solved using a linear programming approach (Dahleh \& Diaz-Bobillo, 1995).

\subsection{Numerical and experimental results}

The design parameters, $\hat{W}_{0}, \bar{w}, \bar{r}$, and $\bar{u}$, are determined by the accuracy of model identification, the inversion algorithm, and the problem of interest. Understanding the effects of these parameters on $\gamma^{*}$ helps in making tradeoff decisions in identification and inversion. In this work $\hat{W}_{0}(\lambda)$ is chosen to be $\frac{1.1759 c_{w}(\lambda-1.0005)}{\lambda-1.1765}$, where $c_{w}>0$ reflects the magnitude of uncertainty. Figs. 13-15 show the dependence of $\gamma^{*}$ on $c_{w}$, $\bar{w}$, and $\bar{u}$. Since the range of $u$ for the case of magnetostrictive actuators is $\left[0, M_{s}^{2}\right]$, parametrizing $\bar{w}$ and $\bar{u}$ in terms of $M_{s}^{2}$ gives a concrete sense on these numbers. From the figures, the higher the uncertainty or the inversion error, the bigger $\gamma^{*}$, and $\gamma^{*}$ drops when $\bar{u}$ is increased until $\bar{u}$ hits $4.5 M_{s}^{2}$, beyond which the saturation constraint no longer plays a role.

From Fig. 15 the tracking performance deteriorates as the saturation constraint $\bar{u}$ is tightened. For the magnetostrictive actuator, $\bar{u}=0.5 M_{s}^{2}$ and strictly enforcing this constraint would lead to large tracking errors. Hence a practical 


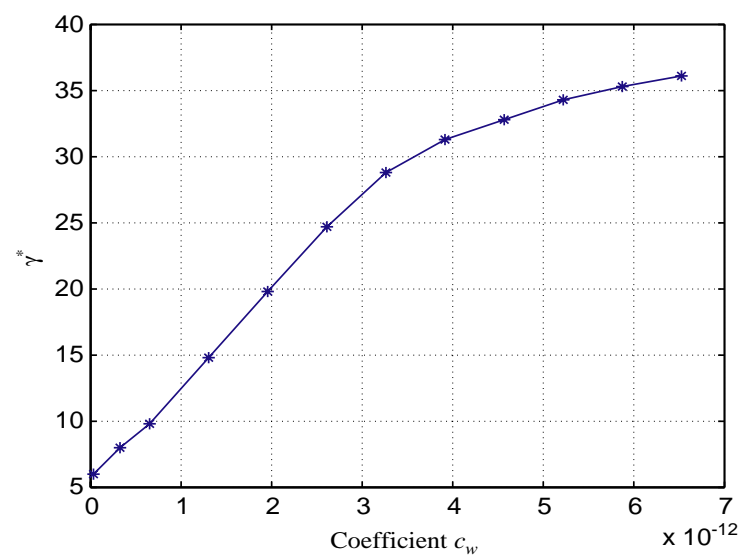

Fig. 13. Effect of the model uncertainty on $\gamma^{*}$. Other parameters: $\bar{r}=30$, $\bar{w}=0.1 M_{s}^{2}, \bar{u}=7.5 M_{s}^{2}$.

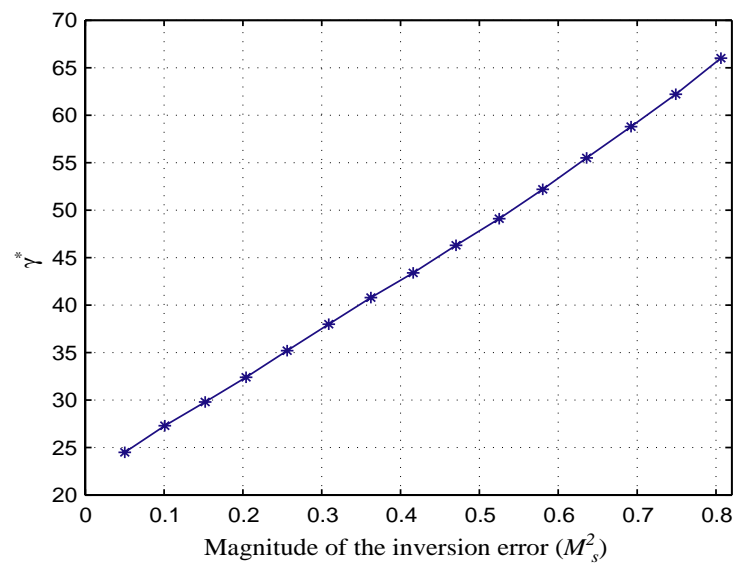

Fig. 14. Effect of the inversion error on $\gamma^{*}$. Other parameters: $\bar{r}=30$, $c_{w}=6.53 \times 10^{-13}, \bar{u}=1.25 M_{s}^{2}$.

approach is to properly relax the constraint. Fig. 16(a) and (b) show the simulation results and the experimental results of tracking a $20 \mathrm{~Hz}$ sinusoidal signal, respectively. In both figures, the measured trajectory and the desired trajectory are in phase, which shows that the controller is effective. Although $\bar{u}$ is relaxed to $3.25 M_{s}^{2}$ in the design, the output $u_{c}$ of $\hat{K}$ stays in the (true) unsaturated region $\left[-0.5 M_{s}^{2}, 0.5 M_{s}^{2}\right]$ except during the transient period (see Fig. 17 for $u_{c}$ in the simulation). The same controller has also been used to track a non-sinusoidal, aperiodic signal (frequency components centering around $30 \mathrm{~Hz}$ ) with a different amplitude range, and it delivers consistent tracking performance (see Fig. 18). This demonstrates the robustness of the controller with respect to reference trajectories.

On the other hand, the tracking errors are relatively large comparing with the results of inverse compensation (Fig. 10(c)). This can mainly be attributed to the saturation constraint (note that in Section $3.2 \bar{y}$ was chosen such that the saturation constraint was automatically satisfied). Fig. 15 reveals a $64 \%$ performance degradation when $\bar{u}$

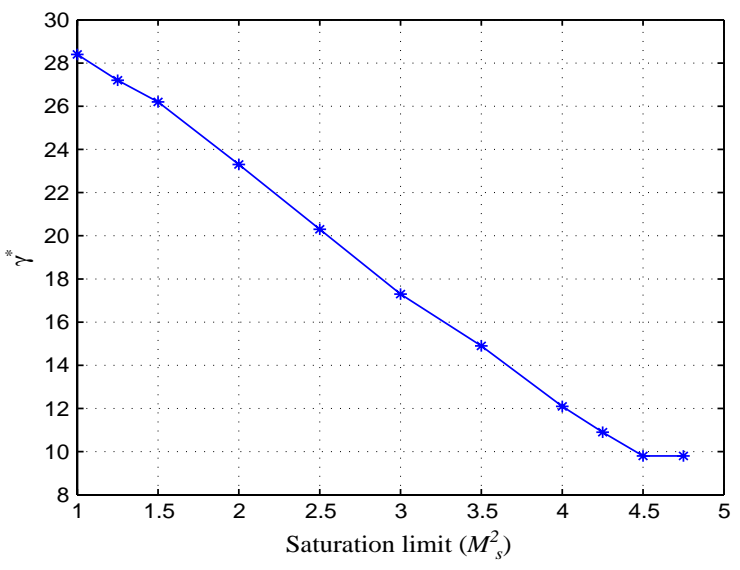

Fig. 15. Effect of the saturation limit on $\gamma^{*}$. Other parameters: $\bar{r}=30$, $c_{w}=6.53 \times 10^{-13}, \bar{w}=0.1 M_{s}^{2}$.
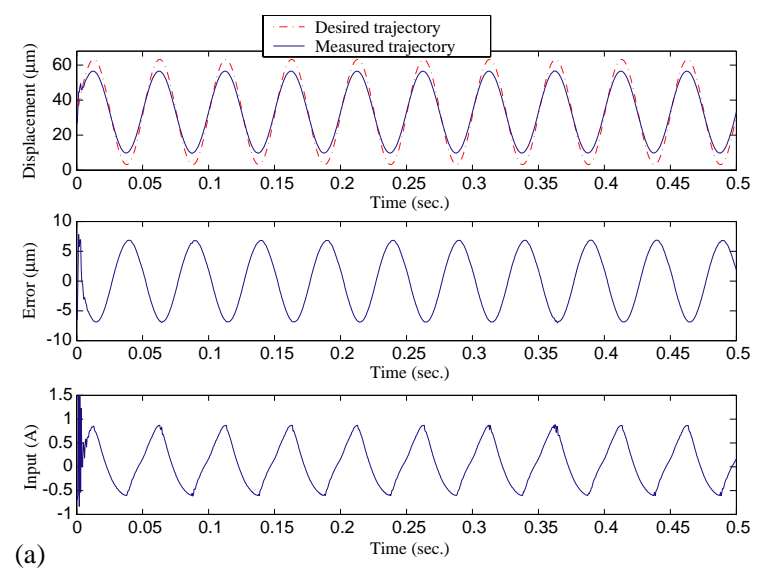

(a)
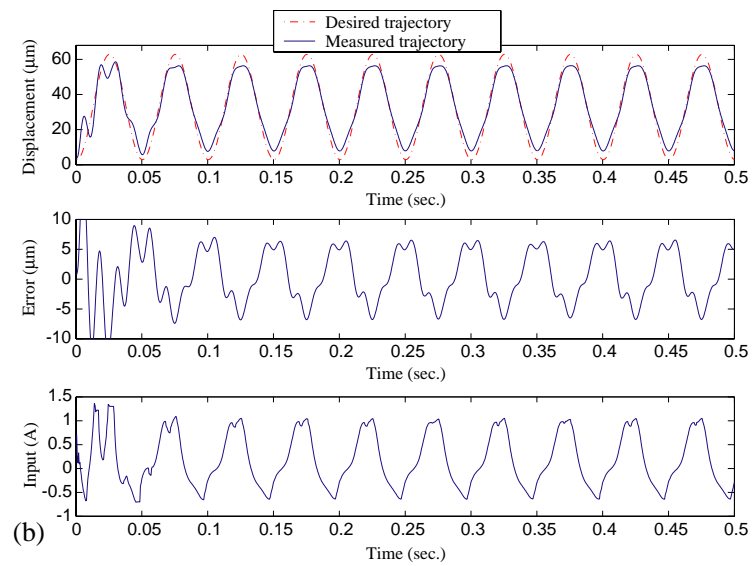

Fig. 16. Results of tracking a sinusoidal signal based on the robust controller ( $\bar{u}=3.25 M_{s}^{2}$ used in the design). (a) Simulation result; (b) Experimental result.

is reduced from $4.5 M_{s}^{2}$ to $3.25 M_{s}^{2}$. The output of $\hat{K}(\lambda)$ is "weaker" than it should be to avoid exceeding the saturation constraint. Unfortunately, $\bar{u}$ cannot be "over-relaxed". Fig. 19 shows that when $\bar{u}$ is relaxed to $5 M_{s}^{2}$ in the design, the tracking performance suffers from persistent saturation of $\hat{K}$ 


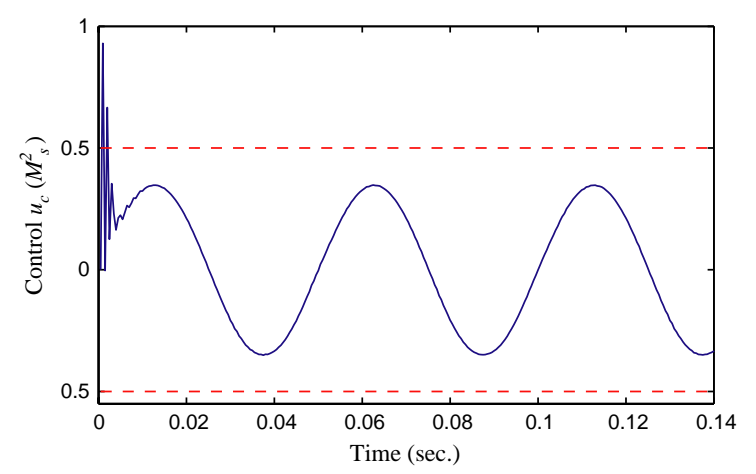

Fig. 17. The output $u_{c}$ of $\hat{K}$ in simulation of tracking the sinusoidal signal.
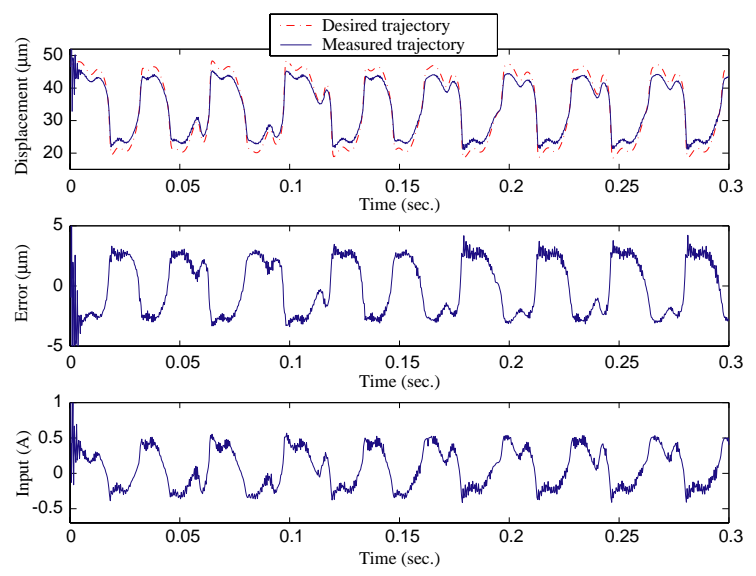

Fig. 18. Experimental results of tracking a non-sinusoidal signal with the same controller as in Fig. 16.
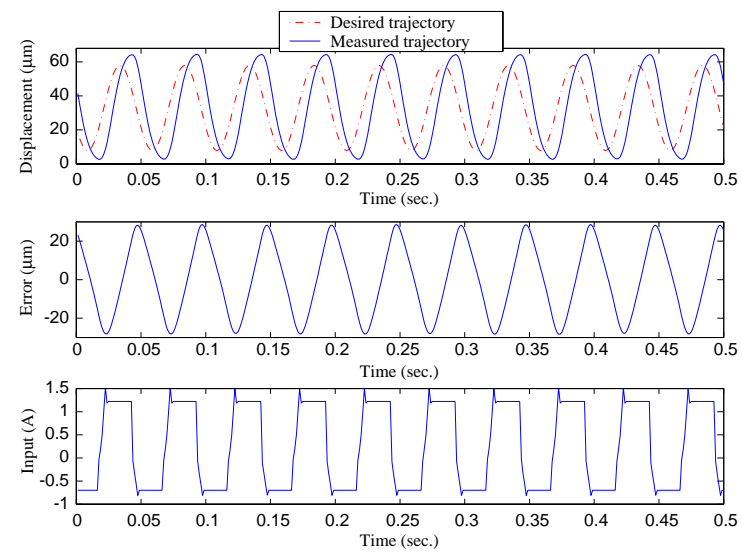

Fig. 19. Experimental results of tracking a sinusoidal signal based on an "over-relaxed"robust controller ( $\bar{u}=5 M_{s}^{2}$ used in the design).

(corresponding to the flat regions of the current input trajectory in Fig. 19). This justifies the necessity of including the saturation constraint in the problem formulation.

\section{Conclusions and Discussion}

In this paper modeling, identification and control of hysteresis in magnetostrictive actuators were studied with the goal of achieving high-bandwidth, full-range operation of these actuators. A novel hysteresis model was proposed together with a parameter identification method and an efficient inverse compensation scheme. Experimental results have demonstrated that the model captures the dynamic and hysteretic behaviors of a magnetostrictive actuator, and that the identification and inverse compensation methods are effective.

To improve the robustness of inverse compensation, a robust control framework was developed by combining the inverse compensation with $l_{1}$ control theory. The reasons to explore $l_{1}$ control (instead of $H_{\infty}$ control) are that the inversion error belongs to $l_{\infty}$ and that the saturation constraint can be appropriately handled. The saturation constraint appears to be a significant performance limiting factor. From the results in Section 4.3, one could improve the tracking performance through improvement of model identification and inversion (smaller $c_{w}$ and $\bar{w}$ ).

Displacement control was the major concern of this paper. As for future work, it would be interesting to extend the results reported here to deal with applications involving the force output of magnetostrictive actuators. Finally it should be noted that some results in this paper (in particular, the inversion algorithm for the Preisach operator and the robust control framework) can be applied to control of a wide class of smart material actuators.

\section{Acknowledgements}

This research was supported by the Army Research Office under the ODDR\& E MURI97 Program Grant No. DAAG55-97-1-0114 to the Center for Dynamics and Control of Smart Structures (through Harvard University) and by the Lockheed Martin Chair endowment funds. The authors wish to acknowledge useful discussions with Professors R. Venkataraman, P. S. Krishnaprasad, M. Brokate, I. D. Mayergoyz, and A. Tits. The authors also wish to thank the anonymous referees for many valuable comments which improved the presentation of this paper.

\section{References}

Adly, A. A., Mayergoyz, I. D., \& Bergqvis, A. (1991). Preisach modeling of magnetostrictive hysteresis. Journal of Applied Physics, 69(8), 5777-5779.

Banks, H. T., Kurdila, A. J., \& Webb, G. (1997). Identification of hysteretic control influence operators representing smart actuators, Part I: Formulation. Mathematical Problems in Engineering, 3(4), 287-328.

Bertotti, G. (1992). Dynamic generalization of the scalar Preisach model of hysteresis. IEEE Transactions on Magnetics, 28(5), 2599-2601. 
Brokate, M., \& Sprekels, J. (1996). Hysteresis and phase transitions. New York: Springer.

Croft, D., Shed, G., \& Devasia, S. (2001). Creep, hysteresis, and vibration compensation for piezoactuators: atomic force microscopy application. Journal of Dynamic Systems, Measurement, and Control, 123(1), $35-43$.

Cruz-Hernandez, J. M., \& Hayward, V. (2001). Phase control approach to hysteresis reduction. IEEE Transactions on Control Systems Technology, 9(1), 17-26.

Dahleh, M. A., \& Diaz-Bobillo, I. J. (1995). Control of uncertain systems: a linear programming approach. Englewood Cliffs, NJ: Prentice-Hall.

Galinaitis, W. S., \& Rogers, R. C. (1998). Control of a hysteretic actuator using inverse hysteresis compensation. In V. Varadan, (Ed.), Mathematics and control in smart structures, Vol. 3323 (pp. 267277). Bellingham, Washington, USA: SPIE.

Ge, P., \& Jouaneh, M. (1996). Tracking control of a piezoceramic actuator. IEEE Transactions on Control Systems Technology, 4(3), 209-216.

Gorbet, R. B., Wang, D. W. L., \& Morris, K. A. (1998). Preisach model identification of a two-wire SMA actuator. In Proceedings of IEEE International Conference on robotics and automation (pp. 2161-2167).

Hughes, D., \& Wen, J. T. (1994). Preisach modeling and compensation for smart material hysteresis. In G.L., Anderson, D.C. Lagoudas, (Eds.), Active materials and smart structures, Vol. 2427 (pp. 5064). Bellingham, Washington, USA: SPIE.

Hoffmann, K.-H., Sprekels, J., \& Visintin, A. (1988). Identification of hysteretic loops. Journal of Computational Physics, 78, 215-230.

Jiles, D. C., \& Atherton, D. L. (1986). Theory of ferromagnetic hysteresis. Journal of Magnetism and Magnetic Materials, 61, 48-60.

Kuhnen, K., \& Janocha, H. (1999). Adaptive inverse control of piezoelectric actuators with hysteresis operators. In Proceedings of European Control Conference (ECC), Karsruhe, Germany, paper F 0291.

Mayergoyz, I. D. (1991). Mathematical models of hysteresis. Berlin: Springer.

Moheimani, S. O. R., \& Goodwin, G. C. (2001). Guest editorial introduction to the special issue on dynamics and control of smart structures. IEEE Transactions on Control Systems Technology, 9(1), $3-4$.

Mrad, R. B., \& Hu, H. (2002). A model for voltage-to-displacement dynamics in piezoceramic actuators subject to dynamic-voltage excitations. IEEE Transactions on Mechatronics, 7(4), 479-489.

Natale, C., Velardi, F., Visone, C. (2001). Modelling and compensation of hysteresis for magnetostrictive actuators. In Proceedings of IEEE/ASME International Conference on Advanced Intelligent mechatronics (pp. 744-749).

Reimers, A., \& Torre, E. D. (1998). Fast Preisach-based magnetization model and fast inverse hysteresis model. IEEE Transactions on Magnetics, 34(6), 3857-3866.

Smith, R. C. (1998). Inverse compensation for hysteresis in magnetostrictive transducers. Technical Report CRSC-TR98-36, CRSC, North Carolina State University.

Tan, X. (2002). Control of smart actuators. Ph.D. thesis, University of Maryland, available as ISR Technical Report PhD 2002-8 at $\mathrm{http}: / /$ techreports.isr.umd.edu/ARCHIVE.

Tan, X., Venkataraman, R., \& Krishnaprasad, P. S. (2001). Control of hysteresis: Theory and experimental results. In V. S. Rao (Ed.), Modeling, signal processing, and control in smart structures, Vol. 4326 (pp. 101-112). Bellingham, Washington, USA: SPIE.

Tao, G., \& Kokotovic, P. V. (1995). Adaptive control of plants with unknown hysteresis. IEEE Transactions on Automatic Control, 40(2), 200-212.

Venkataraman, R. (1999). Modeling and adaptive control of magnetostrictive actuators. Ph.D. thesis, University of Maryland.

Venkataraman, R., \& Krishnaprasad, P. S. (2000). Approximate inversion of hysteresis: theory and numerical results. In Proceedings of the 39th IEEE Conference on Decision and Control, Sydney, Australia, (pp. 4448-4454).
Visintin, A. (1994). Differential models of hysteresis. Berlin: Springer. Webb, G. V., Lagoudas, D. C., \& Kurdila, A. J. (1998). Hysteresis modeling of SMA actuators for control applications. Journal of Intelligent Materials Systems and Structures, 9(6), 432-448.

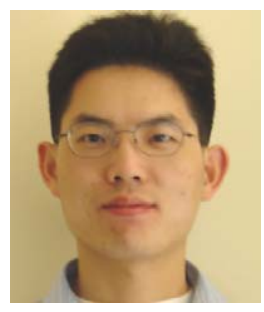

Xiaobo Tan was born in Danyang, China, in 1972. He received the Bachelor's and Master's degrees in Automatic Contorl from Tsinghua University, Beijing, China, in 1995, 1998, and his Ph.D. in Electrical and Computer Engineering from the University of Maryland at College Park, USA, in 2002. His $\mathrm{Ph} . \mathrm{D}$. thesis was focused on modeling and control of hysteresis in smart actuators.

From September 2002 to July 2004 he was a Research Associate with the Institute for Systems Research (ISR) at the University of Maryland. In August 2004 he joined the Department of Electrical and Computer Engineering at Michigan State University as an Assistant Professor. His research interests include modeling and control of smart materials and micro-electromechanical systems, distributed control of networked systems, and numerical integration of dynamical systems on manifolds.

Dr. Tan was an ISR Systems Fellow from 1998 to 2002. He was a finalist for the Best Student Paper Award at the 2002 IEEE Conference on Decision and Control, and a co-recipient of the Best Poster Award at the MEMS Alliance Special Topics Symposium in April 2003.

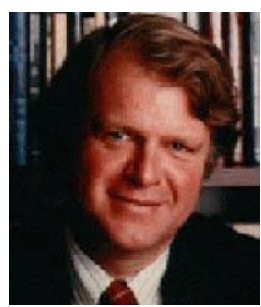

John S. Baras was born in Piraeus, Greece on March 13, 1948. He received the B.S. in Electrical Engineering with highest distinction from the National Technical University of Athens, Greece, in 1970, and the M.S. and $\mathrm{Ph} . \mathrm{D}$. in Applied Mathematics from Harvard University, Cambridge, MA, in 1971 and 1973, respectively. Since 1973 he has been with the Electrical and Computer Engineering Department, and the Applied Mathematics Faculty, at the University of Maryland, College Park, where he is currently a Professor holding a permanent joint appointment with the Institute for Systems Research (ISR). He is also an Affiliate Professor of Computer Science. From 1985 to 1991, he was the Founding Director of the ISR. In February 1990 he was appointed to the Lockheed Martin Chair in Systems Engineering. Since 1991 he has been the Director of the Center for Hybrid and Satellite Communication Networks, a NASA Center for the Commercial Development of Space, which he co-founded. Dr. Baras has held visiting research scholar positions with Stanford, MIT, Harvard University, the Institute National de Reserche en Informatique et en Automatique, and the University of California at Berkeley.

$\mathrm{He}$ has numerous publications in control and communication systems, and is the co-editor of Recent Progress in Stochastic Calculus, Springer, 1990. Dr. Baras hold two patents and has four patent applications pending. His research interests include satellite and hybrid communication networks, integrated network management systems, fast Internet services via hybrid, satellite and wireless networks, network security, stochastic systems, robust control of nonlinear systems, real-time parallel architectures for nonlinear signal processing, intelligent control systems, expert and symbolic systems for control and communication systems synthesis, distributed parameter systems, planning and optimization, real-time architectures for intelligent control, speech and image understanding, biomimetic algorithms and systems for signal processing and sensor networks, intelligent manufacturing of smart materials, integrated product-process design.

Among his awards are: 1978, 1983 and 1993 Naval Research Laboratory Research (Alan Berman) Publication Awards; the 1980 Outstanding Paper Award of the IEEE Control Systems Society; 1991 and 1994 Outstanding Invention of the Year Awards from the University of Maryland; Outstanding Paper Award, "ATM in Hybrid Networks", at Design SuperCon 1996; 1996, MIPS Research Award of Excellence for Outstanding Contributions in Advancing Maryland Industry; 1998, the Mancur Olson Research Achievement Award, from the University of Maryland College Park; 2002, Best paper Award at the 23rd Army Science Conference. 
Dr. Baras is a Fellow of the IEEE. He has consulted extensively with industry and government on various automation, systems and telecommunication problems. He has served in the following: Board of Governors of the IEEE Control Systems Society; IEEE Engineering R\&D Committee; Aerospace Industries Association advisory committee on advanced sensors; IEEE Fellow evaluation committee. He is currently serving on the editorial board of Mathematics of Control, Signals, and Systems, of Systems and Control: Foundations and Applications, of IMA J. of Mathematical Control and Information, of Systems Automation-Research and Applications. 